\title{
First Integral Method: A General Formula for Nonlinear Fractional Klein-Gordon Equation Using Advanced Computing Language
}

\author{
Mohamed A. Abdoon \\ Mathematics Department, Sudan University of Science and Technology, Khartoum, Sudan \\ Email: moh.abdoon@gmail.com
}

Received 25 March 2015; accepted 5 June 2015; published 10 June 2015

Copyright (C) 2015 by author and Scientific Research Publishing Inc.

This work is licensed under the Creative Commons Attribution International License (CC BY). http://creativecommons.org/licenses/by/4.0/

(c) (i) Open Access

\begin{abstract}
In this article, a general formula of the first integral method has been extended to celebrate the exact solution of nonlinear time-space differential equations of fractional orders. The proposed method is easy, direct and concise as compared with other existent methods.
\end{abstract}

Keywords

First Integral Method, Exact Solution, Fractional Klein-Gordon Equation

\section{Introduction}

Partial differential equations arise frequently in the formulation of fundamental laws of nature and in the mathematical analysis of a wide variety of problems in applied mathematics, mathematical physics, and engineering science. This subject plays a central role in modern mathematical sciences. In recent years, it has turned out that many phenomena in fluid mechanics, viscoelasticity, biology, physics, engineering and other areas of science can be successfully modeled by the use of fractional derivatives and integrals [1] [2]. In this article, we study fractional differential equations associated with derivatives. Such a kind of equation appears in many problems. In particular, we have found fractional differential equations related to the classical Klein-Gordon equation [3].

\section{Preliminaries and Basic Definitions and First Integral Method}

The Jumarie’s modified Riemann-Lionvile derivative, of order $\alpha$, can be defined by the following expression 


$$
D_{s}^{\alpha} f(s)=\left\{\begin{array}{l}
\frac{1}{\Gamma(-\alpha)} \int_{0}^{x}(x-\xi)^{-\alpha-1}[f(\xi)-f(0)] \mathrm{d} \xi, \alpha \prec 0 \\
\frac{1}{\Gamma(1-\alpha)} \frac{\mathrm{d}}{\mathrm{d} x} \int_{0}^{x}(x-\xi)^{-\alpha}[f(\xi)-f(0)] \mathrm{d} \xi, 0 \prec \alpha \prec 1 \\
\left(f^{n}(x)\right)^{\alpha-n}, n \leq \alpha \prec n+1, n \geq 1
\end{array}\right.
$$

Moreover, some properties for the modified Riemann-Liouville derivative can be given as follows:

$$
\begin{gathered}
D_{s}^{\alpha} s^{r}=\frac{\Gamma(1+r)}{\Gamma(1+r-\alpha)} s^{r-\alpha}, \\
D_{s}^{\alpha}(f(s) g(s))=f(s) D_{s}^{\alpha} g(s)+g(s) D_{s}^{\alpha} t(s), \\
D_{s}^{\alpha}(f[g(s)])=f_{g}^{\prime}[g(s)] D_{s}^{\alpha} g(s)=D_{s}^{\alpha} f[g(s)]\left(g^{\prime}(t)\right)^{\alpha}
\end{gathered}
$$

Consider the time fractional differential equation with independent variables $X=\left(x_{1}, x_{2}, \cdots, x_{m}, t\right)$ and a dependent variable $u$,

$$
W\left(u, D_{t}^{\alpha} u, u_{x_{1}}, u_{x_{2}}, u_{x_{3}}, D_{t}^{2 \alpha} u, u_{x_{1} x_{1}}, u_{x_{2} x_{2}}, u_{x_{3} x_{3}}, \cdots\right)=0
$$

Using the variable transformation

$$
u\left(x_{1}, x_{2}, \cdots, x_{m}, t\right)=u(\xi), \xi=x_{1}+l_{1} x_{2}+\cdots+l_{m-1} x_{m}+\frac{\lambda t^{\alpha}}{\Gamma(1+\alpha)}
$$

where $k, l_{i}$ and $\lambda$ are constants to be determined later. The fractional differential Equation (5) is reduced to a nonlinear ordinary differential equation

$$
F=\left(u, u_{\xi}, u_{\xi \xi}, u_{\xi \xi}, \cdots\right)=0
$$

We assume that Equation (7) has a solution in the form

$$
u(\xi)=x(\xi)
$$

and introduce a new independent variable $y(\xi)=x^{\prime}(\xi)$,which leads to a new system of ordinary differential equations

$$
\begin{aligned}
& x^{\prime}(\zeta)=y(\zeta) \\
& y^{\prime}(\zeta)=F(x(\zeta), y(\zeta))
\end{aligned}
$$

By using the division theorem for two variables in complex domain $C[x, y]$ which is based on the HilbertNullstellensatz theorem [4], we can obtain a first integral to Equation (9) which can applied to Equation (7) to obtain a first-order ordinary differential equation. An exact solution to Equation (5) is then obtained by solving the equations that are obtained from applying Equation (9) into Equation (7). Now, we wish to quickly recall the division theory.

\section{Theorem 2.1 [The Division Theorem]}

Suppose that $P(x, y)$ and $Q(x, y)$ are polynomials of two variables $x$ and $y$ in $C[x, y]$ and $P(x, y)$ is irreducible in $C[x, y]$. If $Q(x, y)$ vanishes at all points of $P(x, y)$, then there exists a polynomial $G(X, Y)$ in $C[x, y]$ such that $Q(X, Y)=P(X, Y) G(X, Y)$.

\section{The First Integral Method: A General Formula}

We discuss the problem by using the first integral method and consider the general formula [5]:

$$
u^{\prime \prime}(\xi)-s u^{\prime}-k-l u(\xi)-m u^{2}(\xi)-n u^{3}(\xi)-f u^{4}(\xi)-r u^{5}(\xi)=0
$$


where $s, k, l, m, n, f, r$ are real constant.

Substituting Equation (7) in Equation (6), we get the system

$$
\begin{aligned}
& X_{\xi}(\xi)=Y(\xi), \\
& Y_{\xi}(\xi)=s Y+k+I X(\xi)+m X^{2}(\xi)+n X^{3}(\xi)+f X^{4}(\xi)+r X^{5}(\xi)
\end{aligned}
$$

Now, we apply the division theorem. Suppose that $X(\zeta)$ and $Y(\zeta)$ are nontrivial solution of equation (11). Then

$$
q(x, y)=\sum_{i=0}^{M} a_{i}(x) y^{i}=0
$$

which is an irreducible Polynomial in the complex domain $C[X, Y]$, thus

$$
q[X(\zeta), Y(\zeta)]=\sum_{i=0}^{M} a_{i}(X(\zeta)) Y^{i}(\zeta)=0
$$

$a_{i}(X)(i=0,1,2, \cdots, M)$ are polynomial and $a_{M}(X) \neq 0$, Equation (13) is called the first integral method. There exists a polynomial $g(X) X+h(X) Y$ in the complex domain $C[x, y]$ such that

$$
\frac{\mathrm{d} q}{\mathrm{~d} \zeta}=\frac{\mathrm{d} q}{\mathrm{~d} X} \cdot \frac{\mathrm{d} X}{\mathrm{~d} \zeta}+\frac{\mathrm{d} q}{\mathrm{~d} Y} \cdot \frac{\mathrm{d} Y}{\mathrm{~d} \zeta}=(g(X) X+h(X) Y) \sum_{i=0}^{M} a_{i}(X) Y^{i}
$$

which can be written as

$$
\begin{aligned}
& \sum_{i=0}^{M} a_{i}^{\cdot}(X) Y^{i+1}+\sum_{i=0}^{M} i a_{i}(X) Y^{i-1}\left(s Y+k+l X+m X^{2}+n X^{3}+f X^{4}+r X^{5}\right) \\
& =(g(X)+h(X) Y) \sum_{i=0}^{M} a_{i}(X) Y^{i}
\end{aligned}
$$

by comparing the coefficients of $Y^{i}(i=M+1, M, \cdots, 1,0)$ on both sides of Equation (15), we obtain

$$
\begin{gathered}
a_{M}^{\bullet}=h(X) a_{M}(X) \\
a_{M-1}^{\bullet}=g(X) a_{M}(X)+h(X) a_{M-1}(X) \\
\vdots \\
a_{0}^{\bullet}(X)=-2 a_{2}(X)\left(s Y+k+l X+m X^{2}+n X^{3}+f X^{4}+r X^{5}\right)=g(X) a_{0}(X) \\
a_{1}(X)\left(s Y+k+l X+m X^{2}+n X^{3}+f X^{4}+r X^{5}\right)=g(X) a_{0}(X)
\end{gathered}
$$

from (12a), we deduce that $a_{M}(X)$ is a constant and $h(X)=0$, we take $a_{M}(X)=1$, and by balancing the degrees of $g(X), a_{1}(X)$, and $a_{0}(X)$, we find the degree of $g(X)$. Now we consider the following cases.

Case (1)

When $M=1$, in Equation (12), then the Equation (13) becomes

$$
\begin{gathered}
a_{1}^{\bullet}=h(X) a_{M}(X) \\
a_{0}^{\cdot}+s a_{1}(X)=g(X) a_{1}(X)+h(X) a_{0}(X) \\
a_{1}(X)\left(k+l X+m X^{2}+n X^{3}+f X^{4}+r X^{5}\right)=g(X) a_{0}(X)
\end{gathered}
$$

Since $a_{i}(X)(i=0,1)$ are polynomial, then from Equation (14a) we deduce that $a_{1}(X)$ is constant and $h(X)=0$

For simplicity, we take $a_{1}(X)=1$. By balancing the degrees of $g(X)$ and $a_{0}(X)$, we conclude that $\operatorname{deg}(g(X))=2$

Suppose that $g(X)=A_{2} X^{2}+A_{1} X+A_{0}$, then we can find $a_{0}(X)$. 


$$
a_{0}(X)=B_{0}+\left(A_{0}-s\right) X+\frac{A_{1} X^{2}}{2}+\frac{A_{2} X^{3}}{3}
$$

where $B_{0}$ is an arbitrary integration constant. By substituting $a_{0}(X), a_{1}(X)$, and $g(X)$ in Equation (17c), and setting all the coefficients of powers of $X$ to zero, we obtain a system of nonlinear algebraic equations and by solving it, we obtain

$$
r=\frac{1}{3} A_{2}^{2}, f=\frac{5}{6} A_{1} A_{2}, n=\frac{4}{3} A_{2} A_{0}+\frac{A_{1}^{2}}{2}-s A_{2}, m=\frac{3}{2} A_{0} A_{1}-s A_{1}, l=A_{0}^{2}-s A_{0}, k=0, B_{0}=0
$$

Substituting Equation (16) in Equation (10), we obtain

$$
Y=\frac{-2 A_{2} X^{3}-3 A_{1} X^{2}-6\left(A_{0}-s\right) X}{6}
$$

By combining Equation (20) with Equation (11), we find the exact solution of Equation (11).

\section{Case (2)}

When $M=2$, in Equation (15), then the Equation (16) became

$$
\begin{gathered}
a_{2}^{\bullet}(X)=h(X) a_{2}(X) \\
a_{1}^{\bullet}(X)+2 a_{2}(X) s=g(X) a_{2}(X)+h(X) a_{1}(X) \\
a_{0}^{\bullet}(X)+a_{1}(X) s+2 a_{2}\left(k+l X+m X^{2}+n X^{3}+f X^{4}+r X^{5}\right)=g(X) a_{1}(X)+h(X) a_{0}(X) \\
a_{1}(X)\left(k+l X+m X^{2}+n X^{3}+f X^{4}+r X^{5}\right)=g(X) a_{0}(X)
\end{gathered}
$$

Since $a_{2}(X)(i=0,1,2)$ are polynomial, then from Equation (14a) we deduce that $a_{2}(X)$ is constant and $h(X)=0$

For simplicity, we take $a_{2}(X)=1$. By balancing the degrees of $g(X), a_{1}(X)$ and $a_{0}(X)$, we conclude that $\operatorname{deg}(g(X))=2$,

Suppose that $g(X)=A_{2} X^{2}+A_{1} X+A_{0}$, then we find $a_{1}(X)$ and $a_{0}(X)$

$$
\begin{aligned}
a_{1}(X)=B_{0}+\left(A_{0}-2 s\right) X+\frac{A_{1} X^{2}}{2}+\frac{A_{2} X^{3}}{3} \\
a_{0}(X)=B_{1}+\left[A_{0} B_{0}-2 k\right] X+\frac{1}{2}\left[A_{1} B_{0}+A_{0}^{2}-3 A_{0} s+2 s^{2}-2 l\right] X^{2} \\
+\frac{1}{3}\left[A_{2} B_{0}+\frac{3}{2} A_{0} A_{1}-\frac{5 A_{1} s}{2}-2 m\right] X^{3}+\frac{1}{4}\left[\frac{4}{3} A_{0} A_{2}+2 A_{2} s+\frac{A_{1}^{2}}{2}-2 n\right] X^{4} \\
+\frac{1}{5}\left[\frac{5}{6} A_{1} A_{2}-2 f\right] X^{5}+\frac{1}{6}\left[\frac{A_{2}^{2}}{3}-2 r\right] X^{6}
\end{aligned}
$$

where $B_{0}, B_{1}$ are arbitrary integration constants.

By substituting $a_{0}(X) \cdot a_{1}(X)$ and $g(X)$ in (21d), and setting all the coefficients of powers of $X$ to zero, we obtain a system of nonlinear algebraic equations and by solving it, we obtain

$$
k=0, l=\frac{A_{0}^{2}}{4}-\frac{s A_{0}}{2}, m=\frac{9 A_{0} A_{1}}{16}-s A_{1}, n=\frac{4 A_{0} A_{2}+A_{1}^{2}}{8}+\frac{7 s A_{2}}{5}, f=\frac{5 A_{1} A_{2}}{16}, r=\frac{A_{2}^{2}}{12}, B_{1}=0, B_{0}=0
$$

Using Equation (23) in Equation (12), we obtain two equal roots for $Y$.

Note that

$$
a_{0}(X)=\frac{a_{1}^{2}(X)}{4} \text {, then } Y=\frac{-2 A_{2} X^{3}-3 A_{1} X^{2}-6\left(A_{0}-s\right) X}{12},
$$

Combining Equation (24) with Equation (11), we find the exact solution of Equation (11).

\section{Case 3}


When $M=3$, in Equation (15), then the Equation (16) become

$$
\begin{gathered}
a_{3}^{\bullet}(X)=h(X) a_{3}(X) \\
a_{2}^{\bullet}(X)+3 s a_{3}(X)=g(X) a_{3}(X)+h(X) a_{2}(X) \\
a_{1}^{\bullet}(X)+2 s a_{2}(X)+3 a_{3}\left(k+l X+m X^{2}+n X^{3}+f X^{4}+r X^{5}\right)=g(X) a_{2}(X)+h(X) a_{1}(X) \\
a_{0}^{\bullet}(X)+s a_{1}(X)+2 a_{2}\left(k+l X+m X^{2}+n X^{3}+f X^{4}+r X^{5}\right)=g(X) a_{1}(X)+h(X) a_{0}(X) \\
a_{1}(X)\left(k+l X+m X^{2}+n X^{3}+f X^{4}+r X^{5}\right)=g(X) a_{0}(X)
\end{gathered}
$$

Since $a_{2}(X)(i=0,1,2)$ are polynomial, then from Equation (17a) we deduce that $a_{2}(X)$ is constant and $h(X)=0$

For simplicity, we take $a_{2}(X)=1$. Balancing the degrees of $g(X), a_{1}(X)$ and $a_{0}(X)$. We conclude that $\operatorname{deg}(g(X))=2$

Suppose that $g(X)=A_{2} X^{2}+A_{1} X+A_{0}$, then we find $a_{2}(X), a_{1}(X)$ and $a_{0}(X)$ :

$$
\begin{aligned}
& a_{2}(X)=B_{0}+\left(A_{0}-3 s\right) X+\frac{A_{1} X^{2}}{2}+\frac{A_{2} X^{3}}{3} \\
& a_{1}=\frac{1}{6}\left[-3 r+\frac{1}{3} A_{1}^{2}\right] X^{6}+\frac{1}{5}\left[-3 f+\frac{5}{6} A_{1} A_{2}\right] X^{5}+\frac{1}{4}\left[-3 n+\frac{1}{2} A_{1}^{2}+\frac{4}{3} A_{0} A_{2}+\frac{s A_{2}}{3}\right] X^{4} \\
&+\frac{1}{3}\left[-3 m+\frac{3}{2} A_{0} A_{1}-(s+3) A_{1}\right] X^{3}+\frac{1}{2}\left[-3 l+A_{0}^{2}+6 s^{2}-5 s A_{0}\right] X^{2} \\
& a_{0}= B_{1}+\frac{1}{9}\left[\frac{A_{2}^{3}}{18}-\frac{1}{6} A_{2} r\right] X^{9}+\frac{1}{8}\left[\frac{2}{9} A_{1} A_{2}^{2}-\frac{4}{15} f A_{2}\right] X^{8} \\
&+ \frac{1}{7}\left[\frac{7}{18} A_{0} A_{2}^{2}+\frac{7}{24} A_{1}^{2} A_{2}-\frac{5}{12} n A_{2}-\frac{1}{10} f A_{1}+\frac{1}{2} r A_{0}\right] X^{7} \\
&+\frac{1}{6}\left[\frac{1}{2} A_{1} A_{2}^{2}-\frac{2}{3} m A_{2}+\frac{1}{2} A_{0} A_{1} A_{2}+\frac{1}{8} A_{1}^{3}-\frac{1}{4} n A_{1}+\frac{4}{5} A_{0} f\right] X^{6} \\
&+ \frac{1}{5}\left[\frac{5}{6} A_{0}^{2} A_{2}+\frac{1}{2} A_{2}^{2} A_{2}+\frac{1}{8} A_{0} A_{1}^{2}-\frac{7}{6} l A_{2}-\frac{1}{2} m A_{1}+\frac{1}{4} n A_{0}\right. \\
&+\left.\frac{25 s A_{0} A_{2}}{6}+\frac{41 s^{2} A_{2}}{12}+\left(9 n-4 A_{1}\right) s\right] X^{5} \\
&+ \frac{1}{4}\left[-2 l A_{1}-\frac{13 A_{0} A_{1}}{3}+2 m s+\frac{4 s A_{1}}{3}-\frac{l A_{2}}{3}+\frac{A_{0}^{2} A_{1}}{2}\right] X^{4} \\
&+ \frac{1}{3}\left[-\frac{5}{2} l A_{0}+\frac{9 l s}{2}-3 s A_{0}^{2}+\frac{11 s^{2} A_{0}^{2}}{2}+\frac{A_{0}^{2}}{2}-3 s^{2}\right] X^{3}
\end{aligned}
$$

where $B_{0}, B_{1}$ are arbitrary integration constants.

Substituting $a_{0}(X) \cdot a_{1}(X)$ and $g(X)$ in Equation (21d), and setting all the coefficients of powers of $X$ to zero, we obtain a system of nonlinear algebraic equations and by solving it, we obtain

$$
\begin{aligned}
& k=0, l=\frac{A_{0}^{2}}{4}+3 A_{0} s-4 s^{2}, m=\frac{9 A_{0} A_{1}}{16}+\frac{8 s A_{1}}{3}, n=\frac{4 A_{0} A_{2}+A_{1}^{2}}{12}+\frac{A_{2} s}{3}, \\
& f=\frac{5 A_{1} A_{2}}{16}, r=\frac{A_{2}^{2}}{12}, B_{1}=0, B_{0}=0
\end{aligned}
$$

Substituting Equation (23) in Equation (12), we obtain three equal roots for $Y$

Note that 


$$
\begin{array}{r}
a_{1}(X)=\frac{a_{2}^{2}(X)}{3}, a_{0}(X)=\frac{a_{2}^{3}(X)}{27}, \\
\text { then } Y=\frac{-2 A_{2} X^{3}-3 A_{1} X^{2}-6\left(A_{0}-s\right) X}{18},
\end{array}
$$

Combining Equation (24) with Equation (11), we find the exact solution of Equation (11).

By the integrating Equations (20), (25) and (30), we can find different solutions of Equation (10), and the exact solution of the general formula in Equation (10) are given by combining Equations (20), (24), (30), with (11) and integrating respect with respect to $\xi$.

We can apply Theorem 3.1 to studying some time fractional differential equations.

\section{Applications}

\section{The Space-Time Fractional Klein-Gordon Equation [6]:}

Consider the nonlinear fractional Klein-Gordon equation,

$$
\begin{aligned}
& \frac{\partial^{2 \alpha} u(x, t)}{\partial t^{2 \alpha}}=\frac{\partial^{2} u(x, t)}{\partial x^{2}}+a u(x, t)+c u^{3}(x, t), t>0,0<\alpha \leq 1 \\
& u(x, 0)=\sqrt{\frac{a}{c}} \tan \left[\sqrt{\frac{a}{2\left(\lambda^{2}-l^{2}\right)} l x}\right]
\end{aligned}
$$

For our purpose, we introduce the following transformations

$$
u(x, y, t)=u(\xi), \xi=l x+\frac{\lambda t^{\alpha}}{\Gamma(1+\alpha)},
$$

where $l, \lambda$ are constant.

Substituting Equation (33) in Equation (32)

$$
u^{\prime \prime}+\frac{a}{l^{2}-\lambda^{2}} u+\frac{c}{l^{2}-\lambda^{2}} u^{3}=0,
$$

which is the same as in the form of Equation (10) where

$$
\begin{gathered}
s=0, k=0, l=\frac{a}{\lambda^{2}-l^{2}}, m=0, n=\frac{c}{\lambda^{2}-l^{2}}, f=0, r=0 \\
A_{0}= \pm \frac{a}{\sqrt{2 c\left(\lambda^{2}-l^{2}\right)}}, A_{1}=0, A_{2}= \pm \sqrt{\frac{2 c}{\lambda^{2}-l^{2}}} \\
y=\frac{2 A_{0}+A_{1} x^{2}}{2}
\end{gathered}
$$

Integrating with respect to $\xi$ then

$$
\begin{aligned}
& u_{1}(x, t)=\sqrt{\frac{b}{A}} \tan \left(\sqrt{A b}\left(l x-\frac{\lambda t^{\alpha}}{\Gamma(1+\alpha)}+\xi_{0}\right)\right), A>0, b>0 \\
& u_{2}(x, t)=\sqrt{\frac{b}{A}} \tanh \left(\sqrt{A b}\left(l x-\frac{\lambda t^{\alpha}}{\Gamma(1+\alpha)}+\xi_{0}\right)\right), A>0, b<0 \\
& u_{3}(x, t)=\sqrt{\frac{b}{A}} \tan \left(\sqrt{A b}\left(\frac{\lambda t^{\alpha}}{\Gamma(1+\alpha)}-l x+\xi_{0}\right)\right), A<0, b>0
\end{aligned}
$$




$$
u_{4}(x, t)=\sqrt{\frac{b}{A}} \tanh \left(\sqrt{A b}\left(\frac{\lambda t^{\alpha}}{\Gamma(1+\alpha)}-l x+\xi_{0}\right)\right), A<0, b<0
$$

where $A= \pm \frac{a}{\sqrt{2 c\left(\lambda^{2}-l^{2}\right)}}, b= \pm \sqrt{\frac{c}{2\left(\lambda^{2}-l^{2}\right)}}$.

where $\xi_{0}$ is an arbitrary constant.

Consider the initial condition (32), we can see that the generalized the nonlinear fractional Klein-Gordon Equation (32) have the exact solution

$$
u(x, t)=u_{1}(x, t)=\sqrt{\frac{b}{A}} \tan \left(\sqrt{A b}\left(l x-\frac{\lambda t^{\alpha}}{\Gamma(1+\alpha)}+\xi_{0}\right)\right)
$$

Comparing our results with that of resalts [6], shows the novelty of our solution. See Appendix A Figure 1.

\section{Conclusion}

A general formula of the first integral method is used successfully to solve systems of nonlinear fractional differential equations. The performance of this method is reliable, effective and gives more solutions. We have extended the general formula to solve other fractional differential equations.

\section{References}

[1] Feng, Z. (2004) Exact Solutions for the Lienard Equation and Its Applications. Chaos, Soliton \& Fractals, 21, 343-348. http://dx.doi.org/10.1016/j.chaos.2003.10.025

[2] Golmankhaneh, A.K. and Baleanu, D. (2011) On Wave Nonlinear Fractional Klein-Gordon Equation. Signal Processing, 91, 446-451. http://dx.doi.org/10.1016/j.sigpro.2010.04.016

[3] Feng, Z.S. (2002) The First Integer Method to Study the Burgers-Korteweg-de Vries Equation. Journal of Physics A, 35, 343. http://dx.doi.org/10.1088/0305-4470/35/2/312

[4] Zhou, Y.B., Wang M.L. and Wang, Y.M. (2003) Periodic Wave Solution to a Coupled Kdv Equation with Variable Coefficients. Physics Letters A, 308, 31-36.

[5] Abdoon, M. (2015) Programming First Integral Method General Formula for the Solving Linear and Nonlinear Equations. Applied Mathematics, 6, 568-575. http://dx.doi.org/10.4236/am.2015.63051

[6] Ahmed Ali, A. H. and Raslan, K. (2007) New Solutions for Some Important Partial Differential Equations. International Journal of Nonlinear Science, 4, 109-117. 
Appendix A Figure1
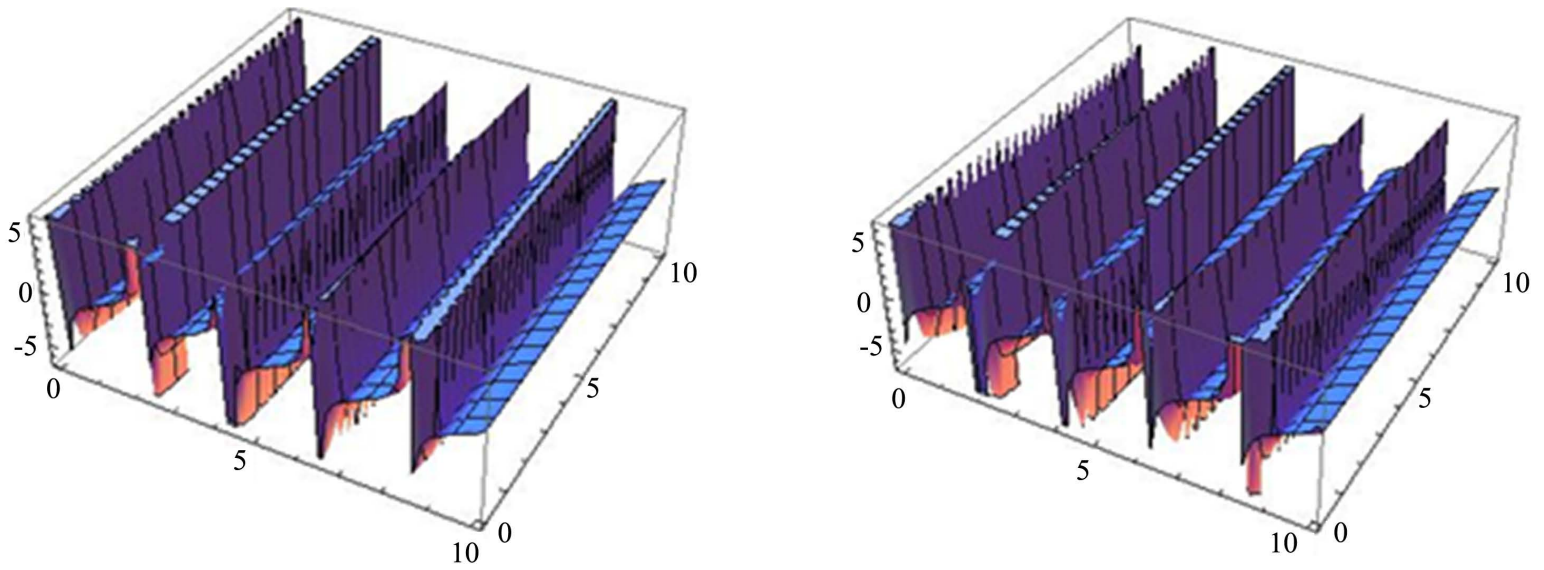

$\alpha=0.01, \lambda=1.9, L=1.5, b=A=1$

$\alpha=0.025, \lambda=1.9, L=1.5, b=A=1$
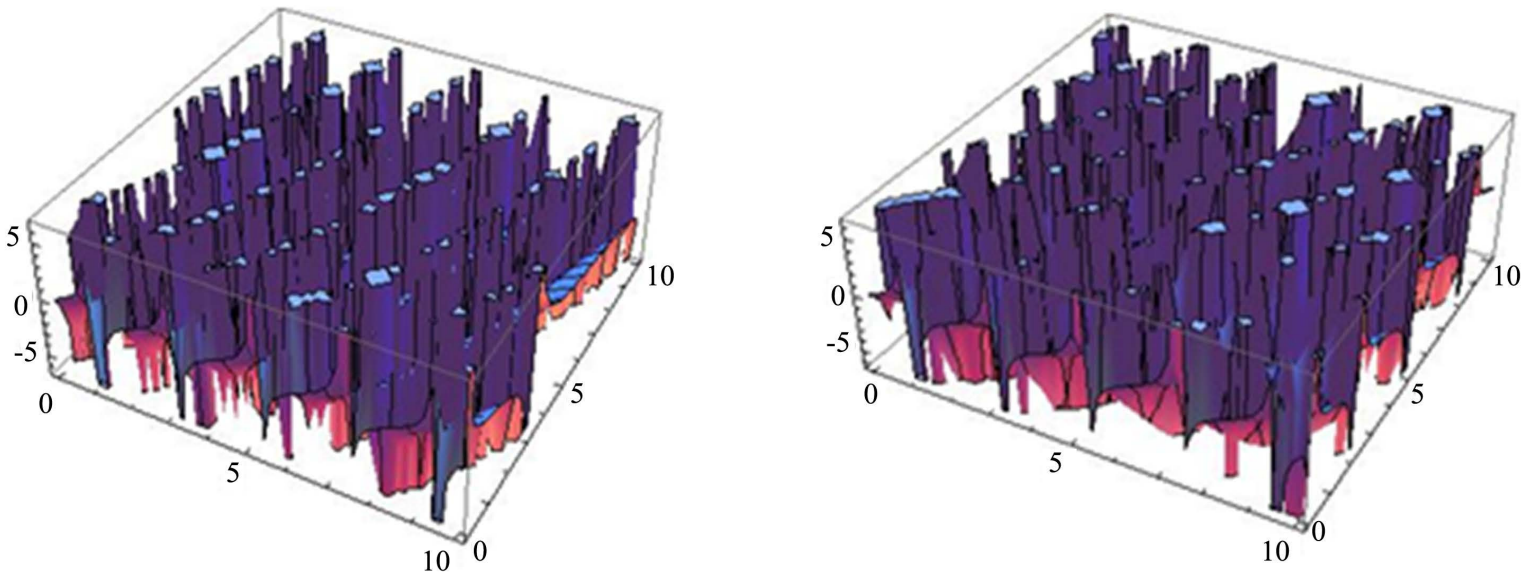

$\alpha=0.5, \lambda=1.9, L=1.5, b=A=1$

$$
\alpha=0.75, \lambda=1.9, L=1.5, b=A=1
$$

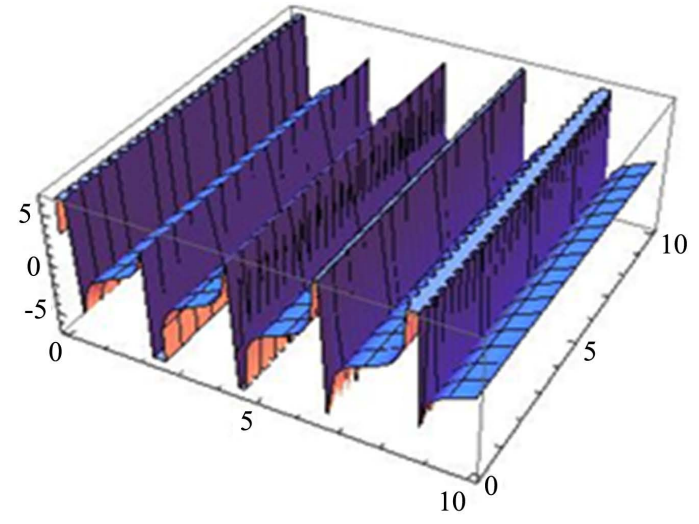

$\alpha=0.0, \lambda=1.9, L=1.5, b=A=1$

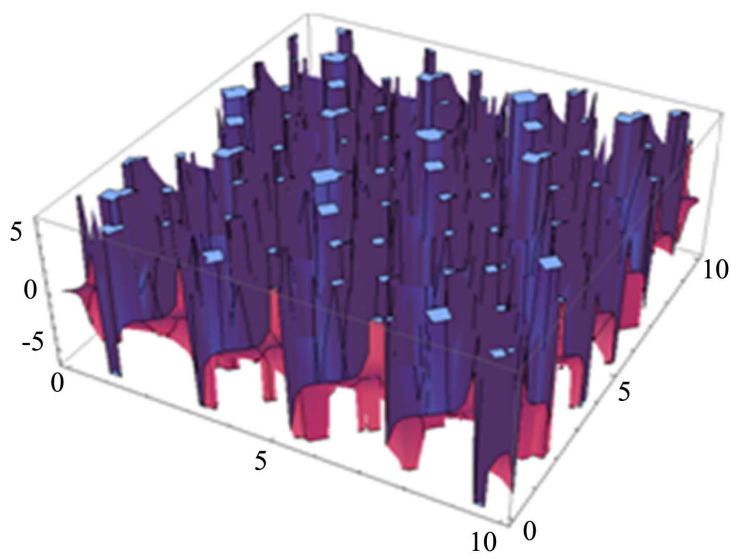

$\alpha=1.0, \lambda=1.9, L=1.5, b=A=1$

Figure 1. Graph of the solution $u(x, t)$ corresponding to the values of $\alpha, \lambda, L$ and $b$ as shown in the caption. 